\title{
Does gravity shape internal representations of space for human 3D perception?
}

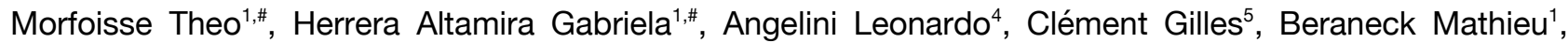 \\ McIntyre Joseph ${ }^{1,2,3}$, Tagliabue Michele ${ }^{1 *}$ \\ \# These authors contributed equally \\ 1 Université de Paris, Integrative Neuroscience and Cognition Center, CNRS UMR 8002, F-75270 Paris, \\ France. \\ 2 Ikerbasque Science Foundation, 48013 Bilbao, Spain \\ 3 Health Division, Tecnalia Research \& Development, E-20009 San Sebastian, Spain \\ 4 HumanTech Institute, University of Applied Sciences Western Switzerland//HES-SO, 1700 Fribourg, \\ Switzerland \\ 5 Lyon Neuroscience Research Center, 69500 Bron. France
}

* Corresponding author: Michele Tagliabue; 45 rue des St-Pères; Université de Paris, Integrative Neuroscience and Cognition Center, CNRS UMR 8002, F-75270 Paris, France. E-mail: michele.tagliabue@parisdescartes.fr

Acknowledgment: The authors thank $M$. Patrice Jegouzo for his technical help in designing the experiments. This work was supported by the Centre National des Etudes Spatiales.

\section{Abstract}

Human visual 3D perception is flawed by distortions, which are influenced by non-visual factors, such as gravitational vestibular signals. Distinct hypotheses regarding the sensory processing stage at which gravity acts may explain the influence of gravity: 1) a direct effect on the visual system, 2) a shaping of the internal representation of space that is used to interpret sensory signals, or 3) a role in the ability to build multiple, modalityspecific, internal depictions of the perceived object. To test these hypotheses, we performed experiments comparing visual versus haptic 3D perception, and the effects of microgravity on these two senses. The results show that visual and haptic perceptual anisotropies reside in body-centered, and not gravity-centered, planes, suggesting an ego-centric encoding of the information for both sensory modalities. Although coplanar, the perceptual distortions of the two sensory modalities are in opposite directions: depth is visually underestimated, but haptically overestimated, with respect to height and width. Interestingly microgravity appears to amplify the 'terrestrial' distortions of both senses. Through computational modeling, we show that these findings are parsimoniously predicted only by a gravity facilitation of cross-modal sensory reconstructions, corresponding to Hypothesis 3. This theory is able to explain not only how gravity can shape egocentric perceptions, but also the unexpected opposite effect of gravity on visual and haptic 3D perception. Overall, these results suggest that the brain uses gravity as a stable reference cue to reconstruct concurrent, modality-specific internal representations of 3D objects even when they are sensed through only one sensory channel. 


\section{Introduction}

Perception in $3 \mathrm{D}$ is a complex phenomenon that includes the ability to determine an item's location in space, as well as its geometrical properties, such as the relative size along each of three dimensions and the relative orientation of its edges. Given its importance for interacting with the physical world, 3D object perception has been deeply investigated. Visual perception has received the most attention, showing how various features of the stimuli as disparities, size, occlusions, perspective, motion, shadows, shading, texture and blur all influence 3D visual perception (Welchman 2016) and how internal models constraint/shape the interpretation of the sensory signals (Curry 1972, Kersten and Yuille 2003, Kersten et al. 2004, Lee 2015).

Despite its critical importance to perception and action, visual perception suffers from measurable distortions: height underestimation with respect to width, also known as the horizontal-vertical, or "L", illusion (Avery and Day 1969, Hamburger and Hansen 2010) and a systematic depth underestimation (Loomis and Philbeck 1999, Todd and Norman 2003). Non-visual factors, such as gravity, also appear to affect visual perception. For example, tilting the body with respect to gravity affects objects recognition (Leone 1998, Barnett-Cowan et al. 2015), orientation and distance perception (Marendaz et al. 1993, Harris and Mander 2014) and other phenomena as the tilted frame illusion (Goodenough et al. 1981, Howard 1982), the oblique effect (Lipshits and Mclntyre 1999, Luyat and Gentaz 2002, Mclntyre and Lipshits 2008) and some geometric illusions (Prinzmetal and Beck 2001, Clément and Eckardt 2005). Furthermore, weightlessness significantly alters the perception of stimulus size and shape, especially in tasks involving depth, during both short-term (Villard et al. 2005, Clément and Bukley 2008, Clément et al. 2008, Harris et al. 2010, Clément and Demel 2012, Clément et al. 2016, Bourrelly et al. 2016) and long-term (Clément et al. 2012-2013, De Saedeleer et al. 2013, Bourrelly et al. 2015) exposure.

One hypothesis to explain gravity-related changes in visual perception is that gravity affects both the eye movements underlying visual exploration (Clément et al. 1986, Reschke et al. 2017-2018) and eye positioning that contribute to the estimation of the visual eye-height, a key reference within the visual scene (Goltz et al. 1997, Bourrelly et al. 2016). Gravity's influence on oculomotor control should specifically affect visual perception, although weightlessness might also induce distinct distortions in other sensory modalities. An alternative hypothesis is that gravity does not affect visual signals per se, but rather it affects an internal representation of space (Clément et al. 2009, 2012) that serves to interpret those signals, independent of the sensory system from which they come (Wolbers et al. 2011, Loomis et al. 2013). A direct implication of this second hypothesis is that microgravity should distort spatial perception in the same way no matter what sensory modality is involved. A third hypothesis proposes that gravity enhances the ability to calibrate and align sensory information coming from different sensory systems (Paillard 1991, Mclntyre and Lipshits 2008, Tagliabue and Mclntyre 2011-2012, 2014, Tagliabue et al. 2013); microgravity might therefore impede the ability to build multiple modality-specific internal representations. Each of these three hypotheses provides a plausible explanation as to why exposure to weightlessness would cause distortions in the 3D perception.

To test these three hypotheses, we first compared distortions of visual versus haptic perception of 3D shape in a normal, upright posture on Earth. Next, we studied the effect of changing the subject's orientation with respect to gravity to assess whether any visual or haptic distortions are egocentric or gravito-centric. Third, we tested the consequences 
of removing the effects of gravity by performing the haptic experiment in parabolic flight, which we compare to similarly-acquired results for vision from the literature. Finally, we performed quantitative simulations to show how the effects of gravity on visual and haptic perception are captured by the hypothesis of multiple, concurrent representations of space aligned, in part, through the use of gravity as a stable reference cue.

\section{Materials and Methods}

In an analogy with previous experiments on visual perception (Clément et al. 2008, 2013), our paradigm was conceptually designed to detect distortions in the perception of a purported 3D cube. The sequential nature of haptic perception induced us, however, to focus each trial on the comparison of the relative size between two out of three possible dimensions. In both the visual and the haptic cases the task consisted of adjusting one side of the rectangle to match the other, so as to form a square. For the haptic tasks, subjects were asked to close their eyes and to feel, through haptic sense only, a rectangular cutout in a rigid, virtual plank generated by a Force Dimension Omega.3 haptic robot (Figure 1A). This manipulandum was able to simulate the presence of a 3D object by applying the appropriate contact forces on the right hand of the subject when he/she performed exploration movements aimed at perceiving its shape and size. During each trial the robot constrained the subject's hand movement to lie within the plane of the virtual plank and to remain inside the rectangle prescribed by the virtual cutout. To allow direct comparisons between the experimental results, an analogous bi-dimensional task was also used for the visual experiments. Subjects were shown a planar rectangle in 3D space, without being able to manually explore it. For trials involving visual perception an Oculus Rift virtual-reality headset was used to show the virtual object. The visual environment was dark and the shapes were represented by light-gray frames. For both sensory conditions the virtual object was located approximatively $40 \mathrm{~cm}$ in front of the right shoulder.

Subjects pressed a button on a trackball when they perceived the object to be square. Although there were no instructions to work quickly, subjects were asked to attempt to perform each trial in a fixed time window (20 s for experiment 1 and 2 and $10 \mathrm{~s}$ for experiment 3). An audible cue indicated to the subject when the end of the allotted time was approaching. The experiment apparatus recorded the subject's responses (dimensions of each rectangle judged to be square) as well as the modification over time of the rectangle's shape as the subject performed the adjustment. For the haptic experiments, the movements of the subject's hand and the contact forces applied against the virtual constraints were also recorded via the haptic device.

The use of bi-dimensional tasks allowed the estimation of the perceptive distortion in one plane at a time. Subjects in our experiments judged the squareness of rectangles lying in each of three anatomical planes: frontal, sagittal or transversal (see bottom part of Figure 1C). The combination of the three possible planes and the two rectangle dimensions resulted in six different geometric configurations that the subject had to deal with. They are represented in the upper part of Figure 2. At the beginning of each trial an audio command told the subject in which anatomical plane the rectangle was lying and which of the two dimensions of the rectangle had to be adjusted. In our paradigm, the reference dimension was always $40 \mathrm{~mm}$, but subjects were not aware of this fact. The initial length of the adjustable side was randomly selected between 15, 25, 35, 45, 55 and $65 \mathrm{~mm}$. Subjects performed five series of trials in all; each series being composed by a random permutation of the six geometric configurations (total number of trials per condition: 30 ). 
A

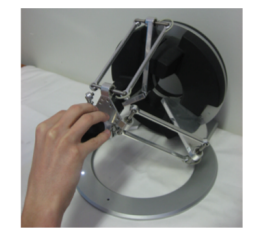

B
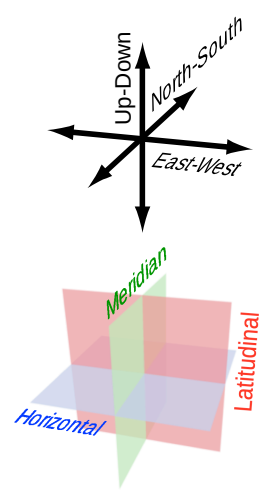

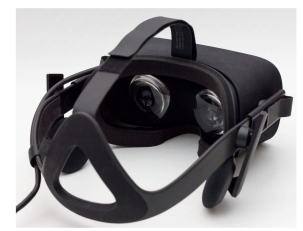

C

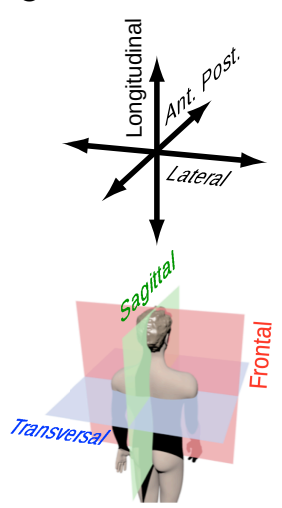

Figure 1: A) Haptic device and virtual reality headset used for the haptic and visual experiments, respectively. In panel $B$ and $C$ are reported the name of the orthogonal directions defined in an external, gravity-centered (UpDown, UD; East-West, EW; NorthSouth, NS) and egocentric, bodycentered (Longitudinal, Lateral and Anterior-Posterior) reference frames respectively. The bottom part of the figure represents the planes in which the task is performed expressed in the gravito-centered (Horizontal, Meridian and Latitudinal) and body-centered (Transversal, Sagittal and Frontal) reference frames.

\section{Experiment 1: Effect of Sensory Modality}

To study the differences and similarities between haptic and visual perception of $3 \mathrm{D}$

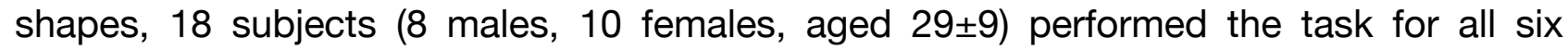
geometrical configurations in each of the two sensory conditions: Haptic and Visual. The order of the two sensory conditions was randomized across subjects.

\section{Experiment 2: Effect of Body Orientation}

To study the perceptive distortions of both haptic and visual senses and whether the information is encoded in an ego-centric (body-centered) or exo-centric (gravity-centered) reference frame, a group of 18 subjects ( 9 male and 9 female, aged $25.5 \pm 5$ years) performed the haptic task in a seated posture (Upright) and lying on the back (Supine), while a second group of 18 subjects ( 11 male and 7 female, aged $24 \pm 4$ years) performed the visual task in the same two postures (Upright and Supine). For the Supine posture subjects laid on a medical bed. The two postures are represented in Figure 2 together with respective correspondence between exo-centric and ego-centric references. The virtual object was placed always at the same distance from the subject's shoulder, independent of the posture. In order to compensate for possible learning effects, the order of the postural conditions was randomized in both sensory conditions. 


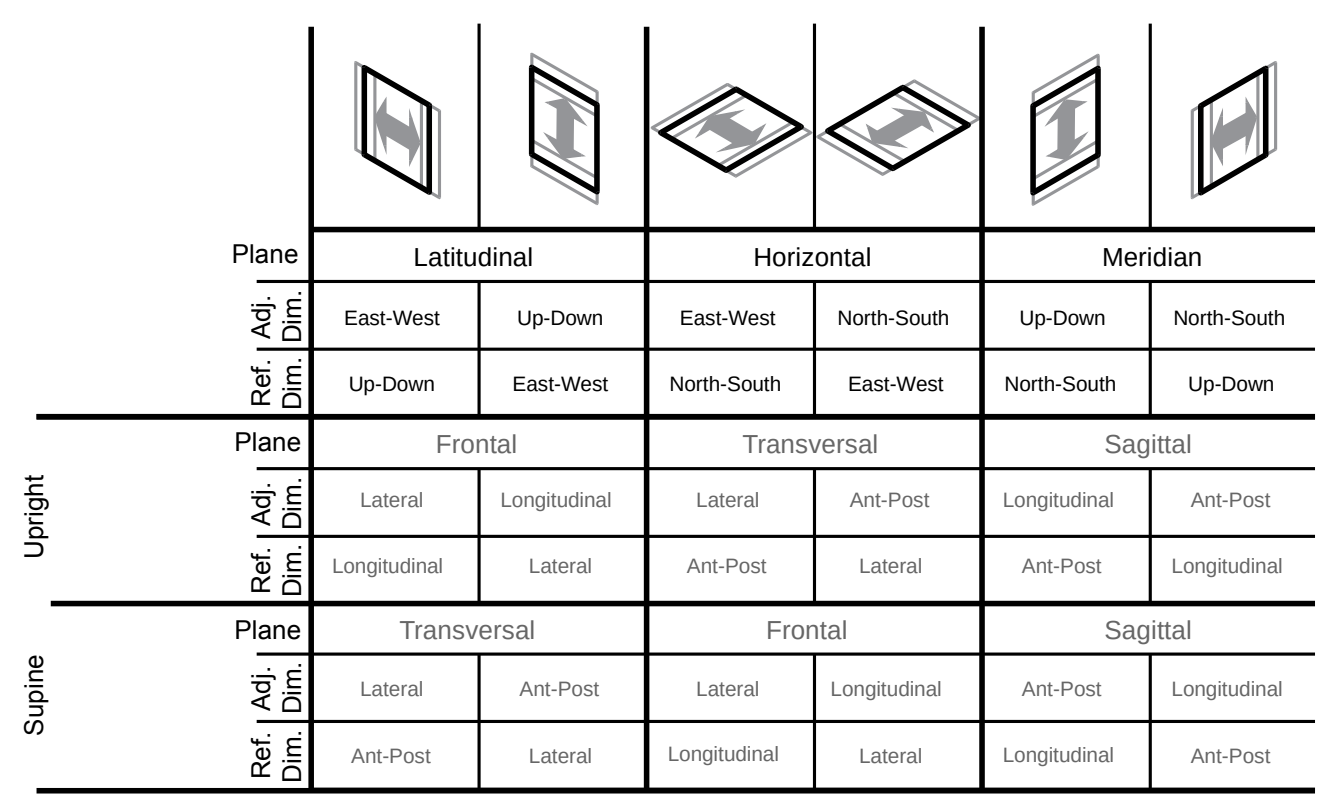

Figure 2: Geometrical Configurations. The first row represents the six geometric configurations, which correspond to the combination of the three planes in which the rectangle could lie and the two different dimensions of the rectangle that the subject had to adjust. The black bold text corresponds to the definitions, in the gravity-centered reference frame, of the task planes, as well as of the adjustable and reference dimensions of each rectangle. Those definitions are independent from the postural condition of Experiments 2 (Upright and Supine) represented in the lower part of the table. For each combination of geometric and postural condition, the table reports with gray bold text the anatomical plane in which the task is performed as well as the anatomical direction of the adjustable and reference dimensions of the rectangles.

\section{Experiment 3: Effect of Weightlessness on Haptic Perception}

To study the role of gravitational cues in the encoding of haptic signals, 18 subjects (10 males, 8 females, aged $38 \pm 11$ years) performed the haptic paradigm in three conditions: normal gravity (1G), during the weightlessness phases of parabolic flight (OG) and in normal gravity but with the arm supported by a strap (Supp). This latter condition was tested to differentiate the biomechanical effect of gravity on the arm from the gravitational stimulation of graviceptors, such as the otoliths.

Parabolic flight provides short intervals $(-20 s)$ of weightlessness within a stable visual environment inside the airplane, bracketed by periods of hyper-gravity $(1.6-1.8 \mathrm{G})$ just before and just after each period of weightlessness. Given the short duration of $0 G$ phases during parabolic flight, the subjects were trained to perform the task in about 10 seconds (two tasks per parabola). Since each subject performed the experiment during 15 consecutive parabolas, he or she could perform all 30 trials per condition.

All experimental conditions were performed inflight onboard of the Novespace Zero-G airplane in order to minimize possible undesired changes in uncontrolled factors. The $1 \mathrm{G}$ and Supp conditions were tested during the steady-flight phase just preceding the first parabola or just following the last parabola of its session, depending on the subject. The subjects were very firmly restrained with belts, so that their relative position with respect to the apparatus did not vary between gravitational conditions. 


\section{Data analysis}

For each trial, $t$, the error, $\varepsilon$, between the length of the adjustable and reference sides of the rectangle was computed. If the exo-centered definition of the three dimensions ( $E W$, $N S$ and $U D$ ) of Figure $1 \mathrm{~B}$ is used, the errors of the six geometric configurations are defined as $E W-U D, U D-E W, E W-N S, N S-E W, U D-N S$ and $N S-U D$, where the minuend and the subtrahend are the adjustable and reference dimensions respectively.

Table 1: Definition of the squaring errors for all six geometrical configurations of the task.

\begin{tabular}{c|c|c|c} 
Plane & $\begin{array}{c}\text { Adjustable } \\
\text { dimension }\end{array}$ & $\begin{array}{c}\text { Reference } \\
\text { dimension }\end{array}$ & Task error \\
\hline \multirow{2}{*}{ Latitudinal } & $\mathrm{EW}$ & $\mathrm{UD}$ & $\varepsilon_{E W-U D}=\varepsilon_{E W}-\varepsilon_{U D}$ \\
\cline { 2 - 4 } & $\mathrm{UD}$ & $\mathrm{EW}$ & $\varepsilon_{U D-E W}=\varepsilon_{U D}-\varepsilon_{E W}$ \\
\hline \multirow{2}{*}{ Horizontal } & $\mathrm{EW}$ & $\mathrm{NS}$ & $\varepsilon_{E W-N S}=\varepsilon_{E W}-\varepsilon_{N S}$ \\
\cline { 2 - 4 } & $\mathrm{NS}$ & $\mathrm{EW}$ & $\varepsilon_{N S-E W}=\varepsilon_{N S}-\varepsilon_{E W}$ \\
\hline \multirow{2}{*}{ Meridian } & $\mathrm{UD}$ & $\mathrm{NS}$ & $\varepsilon_{U D-N S}=\varepsilon_{U D}-\varepsilon_{N S}$ \\
\cline { 2 - 4 } & $\mathrm{NS}$ & $\mathrm{UD}$ & $\varepsilon_{N S-U D}=\varepsilon_{N S}-\varepsilon_{U D}$
\end{tabular}

Table 1 shows how the perceptive distortion associated with each of the three dimensions contributes to the error made on the six geometric configurations. Positive errors correspond to underestimations of the adjustable dimension and/or to overestimations of the reference dimension. Thus, the present experimental paradigm, similar to the one previously used by Clément et al. $(2008,2013)$, allows the quantification of the perceptive distortions of one dimension relative to another, but cannot lead to a measure of the absolute perceptive distortions for each dimension separately (see Annex 1).

\section{Estimation of 3D perceptual distortion}

Table 1 shows that the error in estimating one dimension has opposite effects for the two tasks performed within a given plane. For instance, an overestimation of the NS dimension should result in negative and positive errors in the NS-EW and EW-NS tasks, respectively. These relationships appear to be confirmed by the experimental results (Figure a). It follows that the theoretical relationships below are valid:

$$
\begin{aligned}
& \varepsilon_{E W-N S}=-\varepsilon_{N S-E W} \\
& \varepsilon_{E W-U D}=-\varepsilon_{U D-E W} \\
& \varepsilon_{U D-N S}=-\varepsilon_{N S-U D}
\end{aligned}
$$

Exploiting this property it was possible to combine the five errors obtained for one geometric condition, with the additive inverse of the five errors obtained for the other geometric condition performed in the same plane. This allowed computing the combined mean and the variance of the errors for each of the three planes (Horizontal, Latitudinal and Meridian), instead of individually for each of the 6 geometrical configurations of the task. This technique has the considerable advantage of being more robust, because it is based on 10 samples instead of only 5 . 


$$
\begin{aligned}
& \bar{\varepsilon}_{H o r}=\frac{\sum_{t=1}^{5}\left(\varepsilon_{E W-N S, t}-\varepsilon_{N S-E W, t}\right)}{10} \\
& \sigma_{H o r}^{2}=\frac{\sum_{t=1}^{5}\left(\left(\varepsilon_{E W-N S, t}-\bar{\varepsilon}_{H o r}\right)^{2}+\left(-\varepsilon_{N S-E W, t}-\bar{\varepsilon}_{H o r}\right)^{2}\right)}{10} \\
& \bar{\varepsilon}_{L a t}=\frac{\sum_{t=1}^{5}\left(\varepsilon_{E W-U D, t}-\varepsilon_{U D-E W, t}\right)}{10} \\
& \sigma_{L a t}^{2}=\frac{\sum_{t=1}^{5}\left(\left(\varepsilon_{E W-U D, t}-\bar{\varepsilon}_{L a t}\right)^{2}+\left(-\varepsilon_{U D-E W, t}-\bar{\varepsilon}_{L a t}\right)^{2}\right)}{10} \\
& \bar{\varepsilon}_{M e r}=\frac{\sum_{t=1}^{5}\left(\varepsilon_{N S-U D, t}-\varepsilon_{U D-N S, t}\right)}{10} \\
& \sigma_{M e r}^{2}=\frac{\sum_{t=1}^{5}\left(\left(\varepsilon_{N S-U D, t}-\bar{\varepsilon}_{M e r}\right)^{2}+\left(-\varepsilon_{U D-N S, t}-\bar{\varepsilon}_{M e r}\right)^{2}\right)}{10}
\end{aligned}
$$

With the above formulas one can characterize perceptual distortions in each of three different planes as illustrated in Figure 3. By our convention, a rectangle lying in one of the two vertical planes (Meridian or Latitudinal) is associated with a positive error (stubby rectangle) if the vertical dimension is smaller than the other dimension. In the horizontal plane a positive error (stubby rectangle) corresponds to the depth (NS dimension) being smaller than the width (EW dimension). It is worth noting that if the subject produced a "stubby" rectangle (positive errors) this means that he/she perceived a square to be "slender", and vice versa. The global variance was computed has average of the three planar variances.

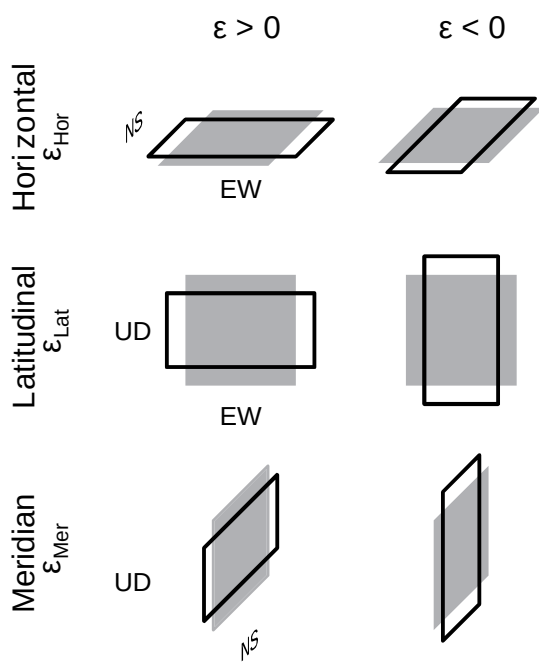

Figure 3: Sign conventions for the errors in the Horizontal, Latitudinal and Meridian planes. The gray squares represent the correct answer (i.e. a square). The black lines represent the distorted answers. Positive planar error values correspond to "stubby" rectangles. Negative values correspond to "slender" rectangle. The same conventions are used for the error expressed in the body-centered planes. In this case Anterior-Posterior, Lateral and Longitudinal directions replace NS, EW and UD respectively. Transversal, Frontal and Sagittal replace Horizontal, Latitudinal and Meridian planes respectively.

We used the same equations to compute the analogous parameter in the ego-centric reference frame after having opportunely replaced the externally defined planes and directions by the body-centered planes (Transversal, Frontal and Sagittal) and directions (Lateral, Anterior-Posterior and Longitudinal) as shown in Figure 2. Table 2 shows the relationships between the planar distortions defined in the body-centered and gravicentered reference frame for the Upright and Supine posture. 
Table 2: Relationship between ego- and exocentrically defined distortions for the Upright and Supine condition.

\begin{tabular}{c|c|c|c} 
Upright & $\bar{\varepsilon}_{\text {Mer }}=\bar{\varepsilon}_{\text {Sag }}$ & $\bar{\varepsilon}_{\text {Lat }}=\bar{\varepsilon}_{\text {Fro }}$ & $\bar{\varepsilon}_{\text {Hor }}=\bar{\varepsilon}_{\text {Tra }}$ \\
\hline Supine & $\bar{\varepsilon}_{\text {Mer }}=-\bar{\varepsilon}_{\text {Sag }}$ & $\bar{\varepsilon}_{\text {Lat }}=\bar{\varepsilon}_{\text {Tra }}$ & $\bar{\varepsilon}_{\text {Hor }}=\bar{\varepsilon}_{F r o}$
\end{tabular}

\section{Reaction forces during haptic task}

To estimate changes of the contact forces between conditions in the haptic tasks, we computed the average components of the reaction forces generated by the haptic device when the subject hand was in contact with the edges of the virtual cutout or when the hand tried to move out of the task plane.

\section{Statistical analysis}

For each experiment we first tested the significance of the distortions by testing for each plane whether the constant errors were on average different from zero (two sided Student's t-test). Then, we performed repeated-measures ANOVA on the constant and variable errors. The sign conventions (Figure 3) being arbitrary, they allow a rigorous comparison of the perceptive distortion within a given plane, but they do not allow the comparison between different planes. For this reason, in the statistical analyses the results on each plane were tested with independent ANOVAs.

Experiment 1: For each of the 3 task planes we tested for an effect of Sensory Modality on the perceptive distortion as a single within-subject independent factor with two levels (Haptic, Visual).

Experiment 2: We tested for an effect of Body Posture as a within-subject independent factor with two levels (Upright, Supine) in separate ANOVAs for each group/sensory modality (Visual and Haptic). Note that this separation is justified by the hypotheses being tested, for which cross effects between posture and modality would have little meaning. To test whether distortions are tied to an ego-centric or gravi-centric reference frame, we defined the task planes both in terms of anatomical axes and world axes. Invariance of distortion (lack of a statistical difference) for the anatomically defined planes, but not the world-defined frames, would indicate that the distortions are primarily egocentrically rather than exocentrically aligned and vice-versa.

Experiment 3: For each of the 3 task planes we tested for an effect of Gravity on haptic perception as a single within-subject independent factor with three levels (1G, 0G, Supported).

Before performing each ANOVA we tested for normality and homogeneity of the distributions using the Kolmogorov-Smirnov and Levenes tests, respectively. To achieve the normal distribution for the response variability, the standard deviations were transformed by the $\log (\sigma+1)$ function (Tagliabue and McIntyre 2011). For the errors expressed in both exocentric and ego-centric reference frames the data were distributed normally (all $p>0.20$ ) and the data variability was similar among all conditions (all $p>0.50$ ).

\section{Quantitative Assessment of Model Predictions}

To adjudicate between three hypotheses that could explain gravity-dependent distortion of shape perception - modality-specific distortion of sensory inputs; distortion of a common, modality-independent internal representation; disruption of sensory motor 
transformations - we performed quantitative simulations of the results predicted by these hypotheses based on distortions and variability of haptically perceived object dimensions measured in Experiment 3 and visually perceived object dimensions from the literature (Clément et al. 2008). The mathematical description of the models are reported in Appendix II.

\section{Results}

\section{Experiment 1: Haptic and Visual perception}

Figure $4 a$ shows that for the six geometric configurations of the squaring task (see methods) the subjects made systematic errors in both visual and haptic conditions. The comparison of the errors made using haptic information alone versus visual information alone shows consistent, opposing results for the two sensory modalities. Hence, in each task, when subjects made on average significant positive errors in the haptic condition, they made negative errors in the visual condition, and vice-versa. Figure $4 \mathrm{~b}$ represents the more robust evaluation of the planar distortions obtained by combining the two sets of squaring tasks performed in the same plane (see methods equation 1 and 2). The amplitude of the distortion was significantly different from zero for both visual and haptic perception in the Sagittal (visual: $F_{(17)}=5.86, p<10^{-4}$, haptic: $F_{(17)}=-9.31, p<10^{-6}$ ) and Transversal plane (visual: $F_{(17)}=-6.66, p<10^{-5}$, haptic: $F_{(17)}=6.82, p<10^{-5}$ ), but was significantly different from zero in the Frontal plane only for the visual tasks (visual: $F_{(17)}=-$ $2.89, p=0.01$, haptic $\left.F_{(17)}=0.09, p=0.92\right)$. Sensory modality had a significant effect in the Sagittal $\left(F_{(1,17)}=75.9, p<10^{-5}\right)$ and Transversal $\left(F_{(1,17)}=65.65, p<10^{-5}\right)$ planes, but not in the Frontal plane $\left(F_{(1,17)}=1.79, p=0.20\right)$. The intra-personal variability of the responses was clearly smaller for the visual modality $\left(\sigma_{\text {hapt }}=6.1 \pm 2.6 \mathrm{~mm}, \sigma_{\text {vis }}=4.2 \pm 2.2 \mathrm{~mm}\right.$, sensory modality effect: $\left.F_{(1,17)}=12.02, p<10^{-2}\right)$.

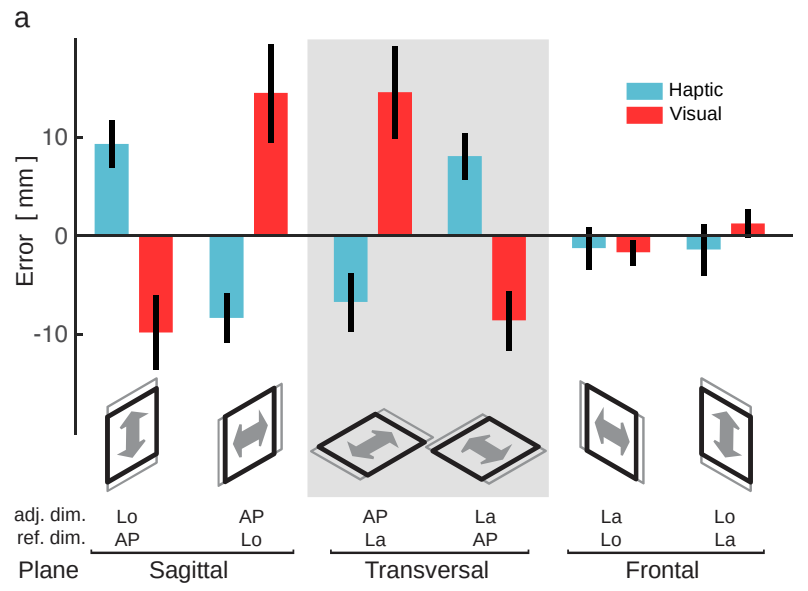

b

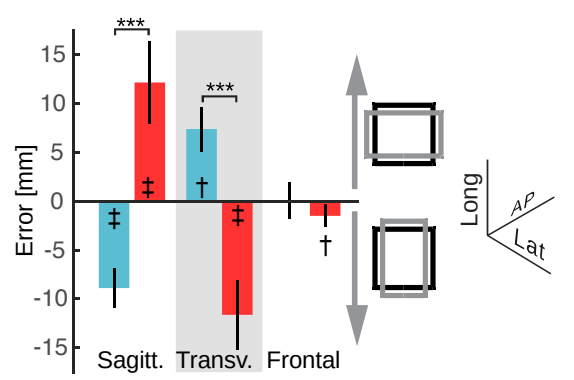

Figure 4: a) Errors for the task performed in each of six geometrical conditions using haptic information only (light blue bars) or visual information only (red bars). Each geometrical condition is characterized by the plane in which the rectangle lies (sagittal, transversal, frontal), and by which direction within the plane was adjustable or held constant: Longitudinal (Lo), Anterior-Posterior (AP) and Lateral (La). Positive errors correspond to the final size of the adjustable dimension being greater than the reference dimension. A significant difference between the two tasks performed in the same plane is indicative of an important perceptive distortion in that specific plane. b) Distortions in the three task planes for haptic and visual conditions. ${ }^{* * *}: p<10^{-3}$ in the ANOVA testing the modality effect. $t$ and $\ddagger$ : $p<10^{-2}$ and $p<10^{-3}$ for the t-test ascertaining differences from zero. c) Illustration of how a cube (gray shape) would be perceived by the subjects when using haptic or visual information alone, respectively. For illustration purposes, the distortions of panel $b$ are scaled up by a factor 5 . 
Remarkably, the significant perceptive distortions in the Sagittal and Transversal planes were in opposite directions between the two sensory conditions. When using haptic(visual) sense, subjects produced rectangles with the Anterior-Posterior dimension smaller(larger) than the Longitudinal and Lateral dimension. The illustration of the perceptive distortion corresponding to the two sensory modalities is reported in Figure 4c. The method used to compute the parallelepiped dimensions is described, with its limitations, in Annex 1.

In summary, Experiment 1 shows clear differences in the patterns of visual and haptic distortions. Distortion appeared primarily in the sagittal and transversal planes and were in opposite direction for the two sensory modalities (contraction and expansion of perceived depth for visual and haptic, respectively).

\section{Experiment 2: Effect of Body Orientation}

The responses of the subjects upright were characterized by constant errors similar to those observed in Experiment 1. The left columns of Table 1 and left panels of Figure 5 show that for both haptic and visual experiments the planar distortions appear consistent between postures if expressed ego-centrically, as we observed no statistically significant effects of posture for any of the three planes when expressed in body-centered reference frame. On the other hand, if the errors are represented in terms of exo-centrically defined planes, i.e. fixed with respect to gravity, a clear effect of posture can be observed in all planes for both sensory modalities (last three columns of Table 1 and right panels of Figure 5).

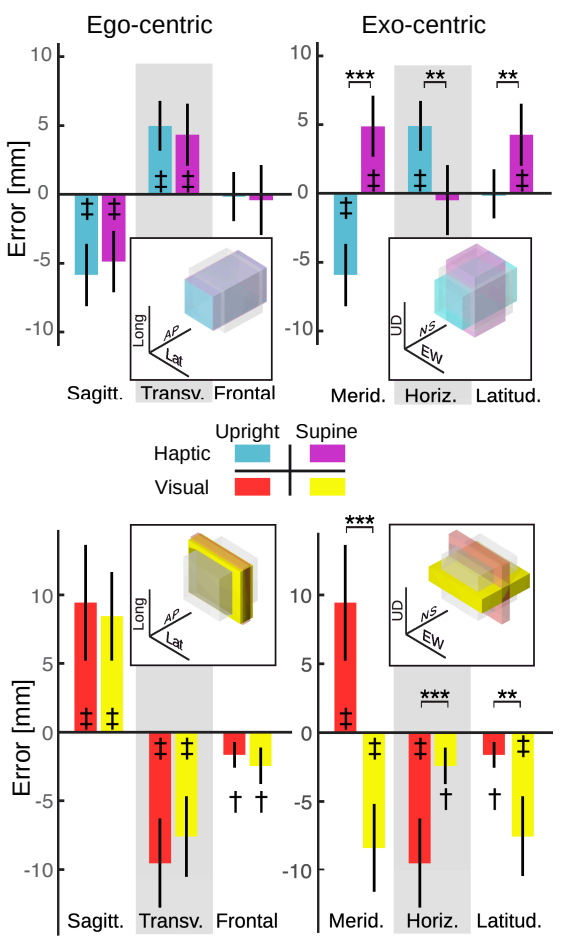

Figure 5: Errors within each plane when the subjects are seated normally (Upright) or lying Supine. The upper and lower panels represent the results for the Haptic and Visual modalities, respectively. The left panels represent the errors per anatomical, ego-centered plane. The right panels represent the data per exo-centered (fixed with respect to gravity) plane. ${ }^{* *}: p<10^{-2}$ and ${ }^{* * *}$ : $p<10^{-3}$ in the ANOVA. + and $\ddagger: p<10^{-2}$ and $p<10^{-3}$ for the t-test ascertaining differences from zero. In each panel the inset illustrates the corresponding $3 D$ perceptive distortion (amplified $\times 5$ ) of a cube.

Table 3: Results of ANOVA for the posture effect on the planar perceptive distortion.

\begin{tabular}{|c|c|c|c|c|c|c|} 
& Sagittal & Transversal & Frontal & Meridian & Horizontal & Latitudinal \\
\hline Haptic & $\begin{array}{c}F(1,17)=0.54 \\
\mathrm{p}=0.47\end{array}$ & $\begin{array}{c}\mathrm{F}(1,17)=0.27 \\
\mathrm{p}=0.61\end{array}$ & $\begin{array}{c}\mathrm{F}(1,17)=0.02 \\
\mathrm{p}=0.90\end{array}$ & $\begin{array}{c}\mathrm{F}(1,17)=37.77 \\
\mathrm{p}<10-4\end{array}$ & $\begin{array}{c}\mathrm{F}(1,17)=12.55 \\
\mathrm{p}=0.002\end{array}$ & $\begin{array}{c}\mathrm{F}(1,17)=8.27 \\
\mathrm{p}=0.010\end{array}$ \\
\hline \multirow{2}{*}{ Visual } & $\begin{array}{c}\mathrm{F}(1,17)=0.88 \\
\mathrm{p}=0.36\end{array}$ & $\begin{array}{c}\mathrm{F}(1,17)=1.65 \\
\mathrm{p}=0.22\end{array}$ & $\begin{array}{c}\mathrm{F}(1,17)=2.46 \\
\mathrm{p}=0.14\end{array}$ & $\begin{array}{c}\mathrm{F}(1,17)=24.76 \\
\mathrm{p}<10-3\end{array}$ & $\begin{array}{c}\mathrm{F}(1,17)=17.85 \\
\mathrm{p}<10-3\end{array}$ & $\begin{array}{c}\mathrm{F}(1,17)=13.36 \\
\mathrm{p}=0.002\end{array}$ \\
\hline
\end{tabular}


The intra-personal variability of the responses was not affected by the posture for the haptic modality $\left(\sigma_{\text {upright }}=6.2 \pm 6.1 \mathrm{~mm}, \sigma_{\text {supine }}=6.6 \pm 6.0 \mathrm{~mm}\right.$, posture effect: $F_{(1,17)}=0.12$, $\mathrm{p}=0.73$ ), but significantly increased in the supine position for the visual experiment $\left(\sigma_{\text {upright }}=3.5 \pm 3.2 \mathrm{~mm}, \sigma_{\text {supine }}=4.8 \pm 4.7 \mathrm{~mm}\right.$, posture effect: $\left.F_{(1,17)}=6.81, p=0.02\right)$.

In conclusion, in this experiment we found that patterns of distortion of both visual and haptic perception were invariant when expressed in an egocentric, but not exocentric reference frame.

\section{Experiment 3: Gravity's Effect on Haptic Perception}

To study the effect of gravity on haptic perception, we start by analyzing the changes in the contact forces between the subject's hand and the virtual object, then the variability/reproducibility of the responses, and finally the pattern of perceptive distortions. The left part of Figure 6A shows that vertical forces applied by the subjects on the upper and lower edge of the sensed object were modulated $\left(F_{(2,34)}=3.9, p=0.02\right)$ by the experimental conditions (1G, OG, Supported). As expected, upward and downward forces increased and decreased respectively in microgravity (post-hoc 1G Vs $0 G, p=0.02$ ), coherent with a reduction of the weight of the upper limb. When the arm weight was supported (see methods), the vertical forces also tended to differ from 1G condition (post-hoc Supp Vs 1G p=0.09) and were modulated in the same direction than in 0G (post-hoc Supp Vs $0 G, p=0.29$ ). Horizontal forces were also significantly affected by the experimental condition $\left(\mathrm{F}_{(2,34)}=6.32, \mathrm{p}<0.01\right.$; Figure $6 \mathrm{~A}$, right panel), with a significant increase of the contact forces in microgravity with respect to the $1 G$ and Support conditions. Globally, the results about the contact forces suggest that the arm support condition successfully mimicked the expected lightening of the arm observed in microgravity. In contrast to the unexpected increase of horizontal contact forces observed in microgravity, arm support did not affect the contact forces in the horizontal direction.

A

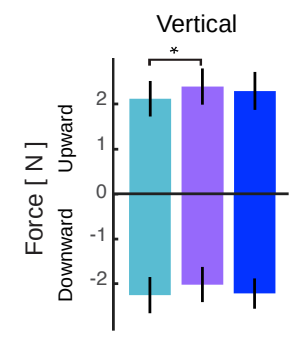

C

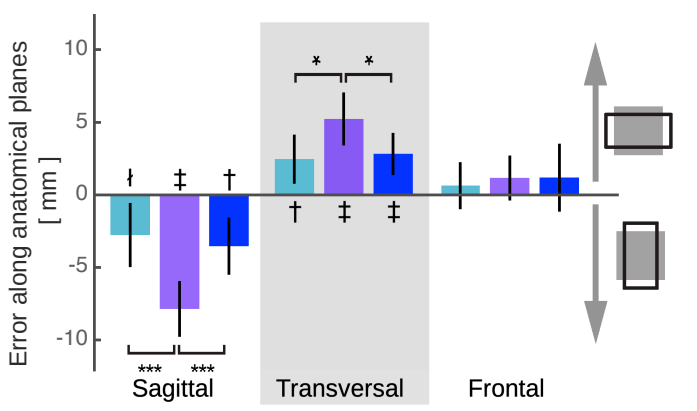

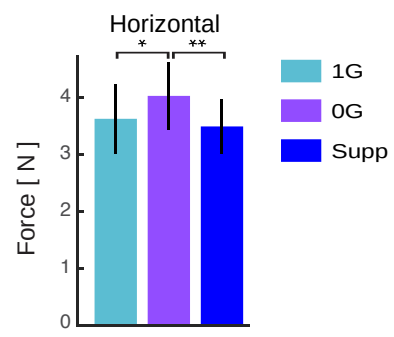

D

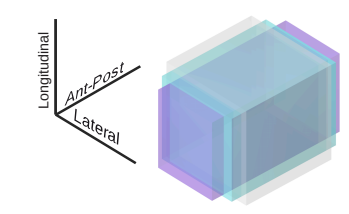

E

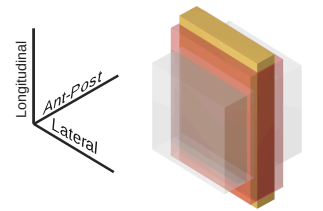

Figure 6: A) Contact forces in the three experimental conditions. Left: Vertical forces generated against the upper and lower edges of the rectangle. Right: Horizontal forces generated against all other edges of the rectangle. B) Within-subject variability of the responses when considering all three planes together. C) Distortions in the three task planes for the three conditions: normogravity $(1 \mathrm{G})$, microgravity $(0 \mathrm{G})$ and with a mechanical support of the arm (Supp). ${ }^{*}: p<0.05$ and ${ }^{* *}: p<10$ 2 in the ANOVA. $\downarrow$, $\dagger$ and $\neq$ : $p<0.05, p<10^{-2}$ and $p<10^{-3}$ for the t-test ascertaining difference from zero. D) Illustration of the haptic perceptive-distortion (experimental results scaled up

a reference cube (gray), in normal gravity (cyan) and in microgravity (violet). E) Illustration of the visual perceptive-distortion (computed from the values of Table 2 in Clément et al. (2008) scaled up by 5) in normal gravity (red) and in microgravity (yellow). 
The precision of responses (response variability in Figure 6B) was not significantly affected by the experimental condition $\left(F_{(2,34)}=1.75, p=0.19\right)$, suggesting that neither microgravity nor the arm support significantly interfered with the subjects ability to perform the task. On the other hand, the comparison of the constant errors, reported in Figure 6C, clearly shows that the perceptive distortion characterizing the Sagittal plane was significantly amplified (became more negative) by microgravity, but was not affected by the arm support (condition effect $\left.F_{(2,34)}=13.65, p<10^{-4}\right)$. Similarly, the distortion in the Transversal plane was amplified (became more positive) in 0G, but was not affected by the support (condition effect $F_{(2,34)}=5.03, p=0.01$ ). Finally, the lack of distortion in the Frontal plane persisted independent of the gravitational and support condition $\left(F_{(2,34)}=0.16, p=0.85\right)$.

To summarize, this microgravity experiment shows that rather than finding a consistent expansion or contraction of 3D space along specific directions common to visual and haptic senses, weightlessness instead amplified the perceptual distortions specific to each modality that were already present in normal gravity. 


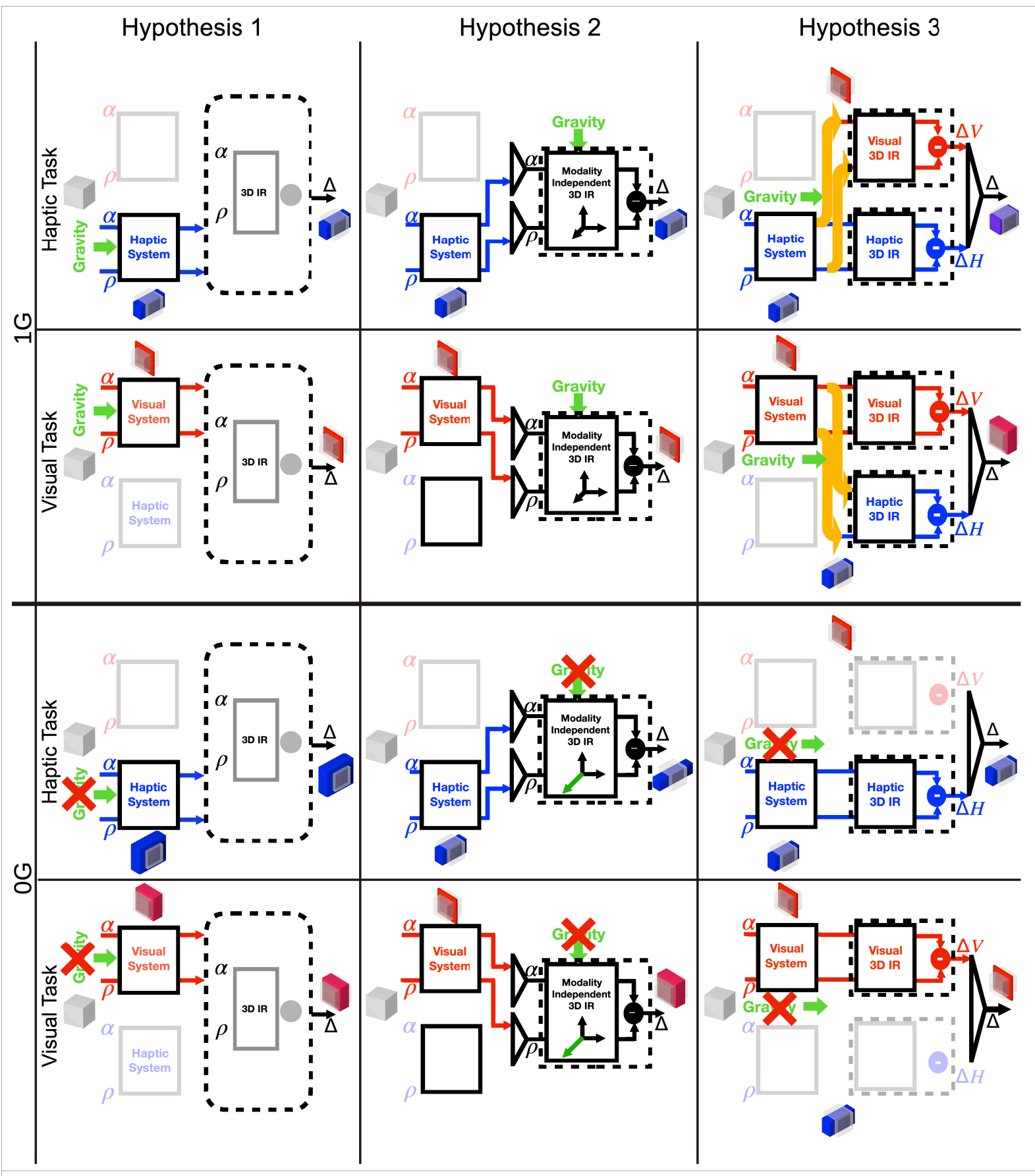

Figure 7: Hypothetical loci of gravity's influence on visual and haptic perception. For all three hypotheses the modality-specific sensory distortions arise from the intrinsic characteristics of the sensory systems. Moreover, the comparison of the size of two perpendicular adjustable $(\alpha)$ and reference $(\rho)$ edges of the object requires the use of an internal representation of the $3 D$ space (3D IR). Normal gravity (1G) and weightlessness (OG) are presented in the upper and lower panel respectively. Hypothesis 1 states that gravity affects the sensory system used to acquire $\alpha$ and $\rho$. These effects are then carried through whatever processing results in the comparison of the two dimensions ( $\Delta)$. Hypothesis 2 states that, independent of the sensory system used to acquire the information, in order to compute the difference between $\alpha$ and $\rho$, the brain reconstructs a modality-independent 3D IR of the object and that gravity affects this latter internal representation, thus influencing the value of $\Delta$. Hypothesis 3 states that, even when the object is sensed through a single sensory modality, the brain reconstructs (yellow arrows) a visual and a haptic internal representation of the 3D object and it compares $\alpha$ and $\rho$ in each of these two sensory modalities separately and concurrently. Only after performing these separate comparisons are the results ( $\Delta V$ and $\Delta H$ ) combined to define the amplitude of the adjustment to be performed ( $\Delta$ ). 


\section{Computational Models}

In an effort to find a theoretical context by which one might understand these results on how gravity affects visual and haptic object perception, we constructed three different computational models according to the three hypotheses alluded to in the introduction and depicted in Figure 7. In all three models the size of the reference $(\rho)$ and adjustable $(\alpha$ ) sides of the object are sensed through the visual or haptic system. To accomplish the task of 'squaring a rectangle' the length of its two edges must be compared ( $\Delta$ is the difference between $\alpha$ and $\rho$ ). This cannot be performed by simply comparing the sensory signals corresponding to two edges, despite the fact that they are both perceived through the same sensory modality. For haptic sense, for instance, tactile exploration of the two edges requires hand movements along perpendicular directions, thus producing very different proprioceptive signals. Similarly, for the visual system, the segments representing the two edges are differently oriented on the retina and their observations requires eyes movements in different directions. As a consequence, the comparison of $\alpha$ and $\rho$ requires first the application of a direction-independent metric, or a mental rotation, which are based on a 3D space internal representation (3D IR). All three hypotheses therefore include some central processing to compare the two edges of the rectangle. All three hypotheses also include gravity, detected through multiple receptors (otoliths, vision, as well as dermal and visceral mechanoreceptors), but differ in terms of where gravity exerts an influence on the tasks studied here, as described in the following paragraphs.

Hypothesis 1: gravity affects directly the encoding of spatial information ( $\rho$ and $\alpha$ ) by the sensorimotor apparatus. For instance, visual perception of depth might be influenced by the orientation of the eye in the orbit that may in turn depend on otolithic signals (Clément et al. 1986, Marendaz et al. 1993, Goltz et al. 1997, Reschke et al. 2017-2018), while haptic perception of movement extent might be affected by gravity-dependent modulation of proprioceptive inputs from the arm due to external forces (Wydoodt et al. 2006). While the model presumes that further central processing is needed to arrive at a comparison $\Delta$ of the two dimensions $\rho$ and $\alpha$, this hypothesis is agnostic to the central processing that occurs once the sensory information has been acquired. Indeed, this hypothesis postulates that gravity exerts an influence before this central processing occurs, whatever it may be. This model is the least restrictive in terms of expected outcomes. Perceptual distortions can be different in each modality and the consequences of removing gravity might also differ in a modality-specific manner.

Hypothesis 2: gravity exerts its influence only after the available sensory inputs have already been integrated into a common, modality-independent representation of 3D space (Wolbers et al. 2011, Loomis et al. 2013), through which the brain interprets sensory signals to reconstruct a 3D representation of the perceived object (Curry 1972 Kersten and Yuille 2003, Kersten et al. 2004, Lee 2015). The sensory inputs associated to $\rho$ and $\alpha$ that feed into this modality-independent representation might be distorted in a modality-specific fashion, but the act of removing gravity would affect only this internal representation, according to this hypothesis. Thus the effect of gravity on the comparison $\Delta$ should be the same no matter which sensory modality is used to accomplish the task. As a result, if weightlessness leads to a dilation of perceived dimensions in depth for the haptic task (i.e. an increase in over-estimation of depth), one would expect a concomitant change of the percept in visual space, i.e. a decrease of the visual underestimation of depth that is observed when gravity is present. 
Hypothesis 3: This hypothesis, eschews the postulate of a common, modalityindependent representation of 3D space in favor of a more distributed computational schema for multimodal integration (McGuire and Sabes 2009, Tagliabue and Mclntyre 2011, 2013, 2014, Arnoux et al. 2017). Under these models, the Central Nervous System (CNS) processes spatial information simultaneously in multiple representations and reference frames linked to each of the underlying sensory apparatuses. The CNS will transform or reconstruct information $(\rho$ and $\alpha)$ in one or more sensor-specific representations based on signals from other modalities. In the present haptic squaring task, the CNS might therefore reconstruct a representation of what the rectangle would look like and then compare the object's dimensions in both haptic and visual space, as if both sensory inputs were available.

One must suppose, however, that transforming spatial representations from one modality to another reproduces the geometrical properties of the target modality as if they were sensed directly, including any spatial distortion that would occur within that modality. As a consequence, when the directly acquired haptic representation of the object and its visual reconstruction are combined, the distortions of the two sensory modalities tend to average out. But what would be the rational for reproducing visual distortion when reconstructing haptic information and vice-versa? The fact that visual and haptic distortions are systematically different on Earth (Experiment 1) implies that when manipulating objects under visual control the brain continuously receives 'conflicting' information. To function correctly the CNS must learn to accommodate these sensory mismatches when spatial information is coming simultaneously from the two sensory systems. Furthermore, previous studies have suggested that multi-sensory integration does not imply multi-sensory calibration, meaning that intermodal conflicts such as the ones that we observed are known to persist (Smeets et al. 2006). To the extent that the CNS must learn intermodal transformations from experience, it follows that, for instance, a stubby haptic percept would be associated with a slender visual percept. For the CNS to be able to use in a like-manner reconstructed information in the place of directlysensed information, the reconstructed information must mimic the spatial properties of the information that it replaces, right or wrong, so that down-stream processing can process it accordingly. It follows that, when reconstructing a visual representation from haptic information, the brain would generate a distorted (slender) object 'image' as if the object would have been seen and not touched, and vice-versa.

Once the comparison has been carried out on both modality-specific representations, the outputs of these two comparisons $(\Delta V$ and $\Delta H)$ would then be combined to give the perception of the overall shape $(\Delta)$. When only one sensory modality is available, as in our experiments, the weight given to the visual and haptic comparisons in the overall output would, however, reflect the fact that a channel that has been reconstructed is less reliable than one that relies on actual sensory inputs. Under this model and the premise that gravity facilitates the reconstruction of sensory information across modalities (Burns and Blohm 2010, Tagliabue and McIntyre 2011-2012, 2014-2015, Tagliabue et al. 2013), the consequences of removing gravity will be to decrease the weight given to information that is not actually sensed (i.e. is reconstructed) thus shifting the response patterns toward the sensory modality that is. Hypothesis 3 predicts, therefore, that, given the opposite distortions of haptic and visual modalities observed in Experiment 1, if distortions in haptic (visual) space are dominant during the haptic (visual) task, those same haptic (visual) distortions will be amplified when the task is performed in 0G. 


\section{Model Predictions}

Qualitatively, one can already surmise that Hypothesis 3 provides a more harmonious explanation of the observed data than either of the other two. Whereas Hypothesis 1 can fully capture the salient features of the experimental outcomes (differing distortions in 1G between the visual and haptic tasks and differing effects of gravity on each of these tasks), it seems unlikely that the apparent amplification of $1 \mathrm{G}$ distortions in $0 \mathrm{G}$, rather than some other effect in another direction, is due to simple chance. It seems more likely that some neural mechanism underlies the fact that $0 G$ distortions appear to remain aligned with $1 \mathrm{G}$ distortions in either modality. And whereas Hypothesis 2 allows for a common effect of gravity on experiments performed in either modality, one would expect those effects to manifest themselves in the same direction (e.g. expansion in depth), regardless of which sensory modality provides the input to the common internal representation. Hypothesis 3 specifically predicts, however, that exposure to weightlessness will shift the overall perception toward the sensory modality that is being sensed at any given time. For the haptic task in weightlessness, since the weight of reconstructed visual representation is reduced, visual distortions modify to a smaller extent the haptic distortions in microgravity. As the 'terrestrial' visual and haptic distortions are primarily in opposite directions (Experiment 1) the result is an amplification of the terrestrial haptic distortions when performing the tasks in 0G (Experiment 3). Symmetrical reasoning can be applied to the case of visual perception to explain the increase of the terrestrially-observed visual distortion when performing the task in microgravity (Clément et al. 2008).

Taking this rationale a step further, we investigated whether Hypothesis 2 or Hypothesis 3 can better reproduce the empirical results at the quantitative level. For Hypothesis 3 we adopt the premise that the weighting between visual and haptic information is determined according to the principles of maximum likelihood (Ernst and Banks 2002), wherein weights are assigned according to the expected variance of signals from different channels, taking into account that "reconstructed" signals will be noisier than their directly-sensed counterparts. The calculations that we used to assign the weights and the underlying assumptions are detailed in Appendix II. With these assumptions the model is characterized by 9 free internal parameters:

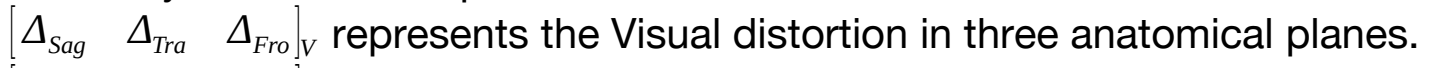

$\left[\begin{array}{lll}\Delta_{\text {Sag }} & \Delta_{\text {Tra }} & \Delta_{F r o}\end{array}\right]_{H}$ represents the Haptic distortion in three anatomical planes.

$\sigma_{\text {met }}^{2}$ is the noise associated to applications of the distance metric or mental rotation.

$\sigma_{t r}^{2}$, is the noise associated to cross-modal transformations in $1 \mathrm{G}$.

$\mathrm{K}$ is the factor by which $\sigma_{t r}^{2}$ increases when gravity is absent.

The simulation consisted of testing whether the model is able to reproduce the experimental results in normal and microgravity on haptic perception presented here in

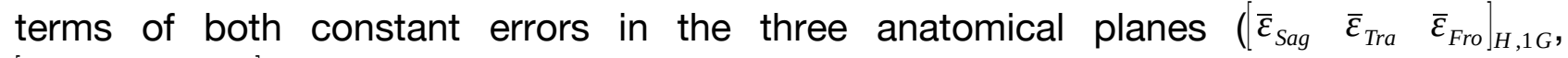
$\left.\left[\begin{array}{lll}\bar{\varepsilon}_{\text {Sag }} & \bar{\varepsilon}_{T r a} & \bar{\varepsilon}_{F r o}\end{array}\right]_{H, 0 G}\right)$ and global variability $\left(\sigma_{H, 1 G}^{2}, \sigma_{H, 0 G}^{2}\right)$; as well as the results reported by Clément et al. (2008) for constant errors of visual perception in the sagittal and transversal planes $\left(\left[\begin{array}{ll}\bar{\varepsilon}_{\text {Sag }} & \bar{\varepsilon}_{\text {Tra }}\end{array}\right]_{V, 1 G},\left[\begin{array}{ll}\bar{\varepsilon}_{\text {Sag }} & \bar{\varepsilon}_{\text {Tra }}\end{array}\right]_{V, 0 G}\right)$.

Hypothesis 2 can be represented mathematically by the equations:

$$
\begin{aligned}
& {\left[\begin{array}{lll}
\Delta_{\text {Sag }} & \Delta_{\text {Tra }} & \Delta_{\text {Fro }}
\end{array}\right]_{H, 0 G}=\left[\begin{array}{lll}
\Delta_{\text {Sag }} & \Delta_{\text {Tra }} & \Delta_{\text {Fro }}
\end{array}\right]_{H, 1 G}+\left[\begin{array}{lll}
\Delta_{\text {Sag }} & \Delta_{\text {Tra }} & \Delta_{\text {Fro }}
\end{array}\right]_{\text {met }, 0 G}} \\
& {\left[\begin{array}{llll}
\Delta_{\text {Sag }} & \Delta_{\text {Tra }}
\end{array}\right]_{V, 0 G}=\left[\begin{array}{lll}
\Delta_{\text {Sag }} & \Delta_{\text {Tra }}
\end{array}\right]_{V, 1 G}+\left[\begin{array}{ll}
\Delta_{\text {Sag }} & \Delta_{\text {Tra }}
\end{array}\right]_{\text {met }, 0 G}}
\end{aligned}
$$



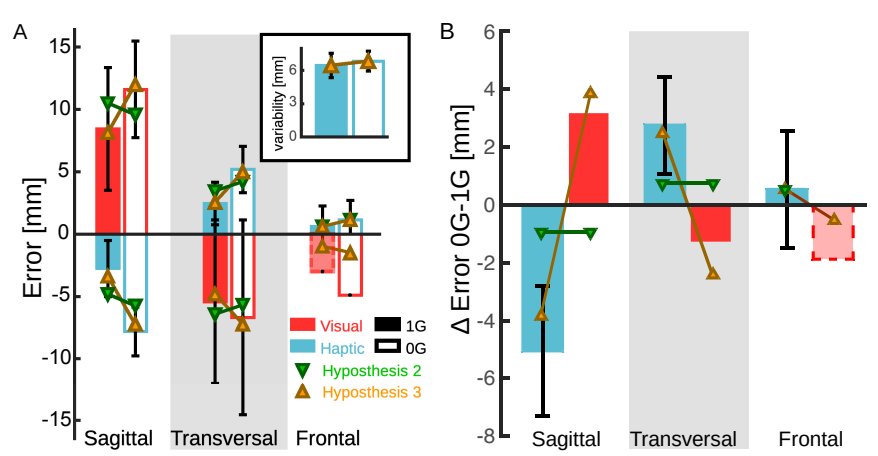

Figure 8: A) Comparison between the experimental data and the model predictions of the distortion in the three task planes. Bars correspond to the average ( \pm confidence interval) experimental results. Light blue bars correspond to the haptic task (Experiment 4). Red bars correspond to experimental results obtained by Clément et al. (2008) for the visual task in Sagittal and Transversal planes. Data for visual distortion in the Frontal plane (pink) have been indirectly computed from the results reported by Clément et al. (2008) by invoking the chain rule. The inset represents the global within-subject variability of the responses in the haptic task. B) The comparison between experimental data and model predictions is represented in terms of the effects of the gravity (0G-1G data) on the haptic and visual distortions for the three anatomical planes.

characterized by 8 free parameters: the haptic ( $\left.\Delta_{\text {Sag }} \Delta_{\text {Tra }} \Delta_{F r o}\right]_{H, 1 G}$ ) and visual (

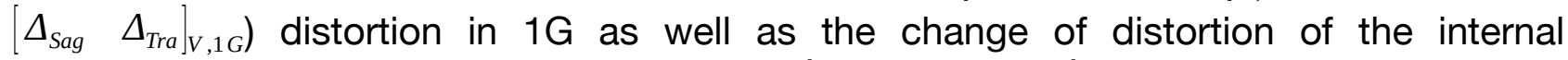
representation of space due to microgravity $\left(\left[\begin{array}{lll}\Delta_{\text {Sag }} & \Delta_{\text {Tra }} & \Delta_{\text {Fro }}\end{array}\right]_{\text {met }, 0 G}\right)$. This model makes no prediction about response variability, therefore the results can be compared to the experimental data in terms of constant errors only.

Figure 8 shows that the model representing a distortion of the internal representation of space is not able to predict the experimentally observed increase of the "terrestrial" haptic (Experiment 3) and visual (Clément et al. 2008) distortions in microgravity. On the other hand, the concurrent model (Hypothesis 3) is able not only to predict the experimentally observed modulation of the haptic and visual distortions, but also the response variability in the haptic task. Therefore, among the three hypothetical mechanisms proposed to explain the effect of gravity in spatial perception, a role in cross-modal sensory transformations emerged as the most likely explanation of the empirical results reported here and elsewhere.

The fitted model parameters used to produce these results are reported in Table 3 . The fact that values for $\sigma_{m e t}$ and $\sigma_{t r}$ are higher than the estimates used to parameterize the sensory inputs $\left(\sigma_{H}=3 \mathrm{~mm}, \sigma_{V}=1.5 \mathrm{~mm}\right)$ are consistent with the idea of an important role of 3D "mental rotation" and cross-modal sensory transformation in the performance of the tasks. The $\mathrm{K}$ factor significantly larger than 1 corresponds to a significant increase in microgravity of the noise associated to the cross-modal transformations.

Table 4. Optimal value of the free parameters of the "Concurrent Representation Model": variability associated to the $3 \mathrm{D}$ metrics $\left(\sigma_{\text {met }}\right)$ and to the cross-modal sensory transformations $\left(\sigma_{t r}\right)$, the amplification factor $(K)$ of the noise associated to the sensory transformations in microgravity and the perceptive distortions $\Delta$ associated to the visual $(V)$ and haptic $(H)$ sense, for the three body-centered planes (Sagittal, Transversal and Frontal).

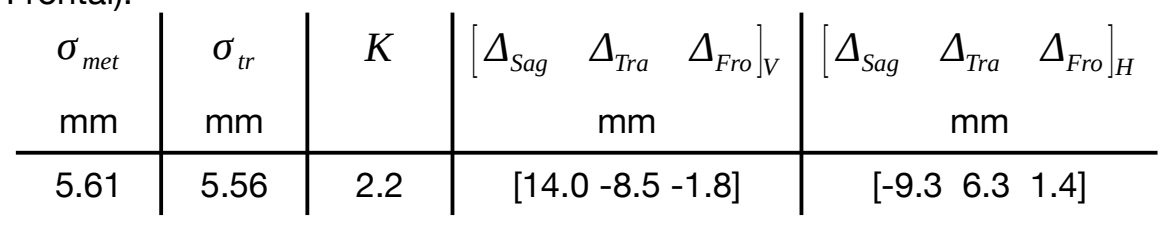




\section{Discussion}

The experiments presented here aimed to understand how gravity affects perception of 3D geometry. Previously restricted to vision (Clément et al. 2008, 2013, Clément and Reschke 2010), we extend these studies to include haptic sensation. In the following, we discuss each of our empirical results with respect to reports in the literature, followed by a theoretical discussion on gravity's role in the perception of three-dimensional space.

\section{Haptic Vs Visual perception}

Experiment 1 revealed very clear differences between haptic and visual perception of 3D geometry. Subjects visually underestimated an object's depth with respect to both height and width, whilst overestimating depth when exploring the object haptically.

Individually, the visual and haptic distortions observed here are consistent with previous findings. Our results correspond well to overestimation in the radial dimension observed for haptic tasks performed in the horizontal plane (Lipshits et al. 1994, Armstrong and Marks 1999, Fasse et al. 2000, Henriques and Soetching 2003). Initially ascribed to different velocities of the explorative movements in the radial and tangential directions (Armstrong and Marks 1999), more recent studies have rejected this explanation (McFarland and Soetching 2007). To our knowledge no previous data exists about the haptic perception of object dimensions in the sagittal and frontal planes. Similarly, the underestimation of depth for vision corresponds well to what was previously observed in the horizontal plane (Wagner 1985, Loomis and Philbeck 1999). Surprisingly, we observed no significant distortion in the frontal plane, as one might have expected given the wellknown horizontal-vertical, or "L", illusion (Avery and Day 1969, Hamburger and Hansen 2010). That the object was situated in front of the right shoulder, rather than straight ahead, might explain this discrepancy, as the vertical line would less likely be associated to depth (Girgus and Coren 1975).

Collectively, our results represent to our knowledge the first direct comparison of visual and haptic perception of 3D shape. Although perceptual biases were known to differ between visually and haptically guided pointing, the analysis of this motor tasks could not show that the perceptive errors are in opposite directions with respect to the actual target position (vanBeers et al 1999, Liu et al. 2018). Only one study, limited to a single degree of freedom, showed opposite visual and proprioceptive errors consistent with our findings (Goble and Brown 2008). Our empirical observations of opposing visual and haptic distortions represent, therefore, a novel finding on which is based our interpretation of how gravity affects 3D perception, to be discussed below.

\section{Effect of Body Orientation}

The comparison of seated versus supine body orientation clearly showed that both visual and haptic distortions align with the subject's body rather than with gravity. This is in line with findings about body-centered haptic perception (Gurfinkel et al. 1993, Dupin et al. 2018) and about eye-centered visual encoding of object shape (Howard et al. 1990, Averly and Day 1969) and position (Mclntyre et al. 1997, Henriques et al. 1998, Vetter et al. 1999, Buneo et al. 2002). This is nevertheless somewhat surprising given the effects of body orientation on a variety of visual tasks (Goodenough et al. 1981, Marendaz et al. 1993, Leone 1998, Prinzmetal and Beck 2001, Clément and Eckardt 2005, Dyde et al. 2006, Harris and Mander 2014, Barnett-Cowan et al. 2015). 
Our results about haptic perception while lying supine represent a novel dataset. The only previous experiments known to us focused on the haptic perception of orientations, where gravity does seem to have an effect (Luyat et al. 2001, Lejeune et al. 2004, Mclntyre and Lipshits 2008, Gentaz et al. 2008). Given that forces applied to the exploring hand affect haptic perception of distances (Wydoodt et al. 2006), and that tilting the subject changes the anatomical direction of the forces due to the weight of the arm, one might predict an effect of posture on haptic perception of 3D shape. It is therefore surprising that we saw no changes in haptic distortion between an upright and supine posture. The invariance of these ego-centered distortions suggests that the brain might compensate for changes of gravitational loads, probably using otolithic or other signals to detect the direction of gravity.

\section{D Perception in Weightlessness}

We observed a clear increase of perceptive distortions in the sagittal and transversal planes in 0G, which happen to be the same two planes in which the perceptive distortions are significant in normogravity. The invariance of the haptic distortions when the subject was supine and when the subject's arm was artificially supported suggests that the effects of microgravity are not directly ascribable to the mechanical action of gravity on the arm. Nor does it seem to be due to a decrease in the precision of haptic sensation, because response variability did not change in microgravity. This is in contrast to a decrease of proprioceptive precision previously observed in weightlessness (for a review see Clément and Reschke 2010). This latter result was, however, linked to a decrease in muscle tension in 0 G, while we observed no 0G-related decrease in contact forces during haptic exploration. We can therefore assume that muscle tone and the precision of proprioceptive signals were similar between $0 \mathrm{G}$ and $1 \mathrm{G}$ in our experiments. One might even speculate that the increase of the contact force exerted by the subjects to be part of an active strategy aimed at maintaining good performance in microgravity. The most remarkable result from our microgravity experiments, however, is that weightlessness increases over-estimation of depth with respect to width and height for haptic perception (Experiment 3), compared to the previously observed increase in underestimation of depth in the visual domain (Clément et al. 2008).

\section{How does gravity affect 3D perception?}

Both visual and haptic 3D perception appear to be ego-centric (Experiment 2) but affected by the lack of gravity (Experiment 3, Clément et al. 2008, 2013). These findings appear contradictory. How can an external cue, such as gravity, affect sensory signals encoded in eye- or body-centered reference frames, which are theoretically independent of external information?

A first explanation might be that gravity acts directly on the sensory systems (Hypothesis 1). The fact that weightlessness appears to affect visual and haptic distortions in the same planes (sagittal and transversal), but in opposite directions makes this explanation unlikely. It is improbable that gravity affects two independent sensory systems precisely in the same two planes and systematically in opposite directions. Moreover, the observed effect of weightlessness on haptic perception cannot be ascribed to the biomechanical effect of gravity on the sensing arm, as noted above.

A second line of reasoning (Hypothesis 2) is that gravity affects a modality-independent representation of space (Wolbers et al. 2011, Loomis et al. 2013), as has been proposed to explain the effects of otolithic deficits (Clément et al. 2009) and long-duration exposure to microgravity (Clément et al. 2012) on visual illusions. If this were the case, then 
weightlessness should distort both visual and haptic perceptions in the same way. The opposing effects of microgravity on haptic (Experiment 3) versus visual (Clément et al. 2008) perception suggest that Hypothesis 2 should also be rejected.

Here we have proposed a third explanation based on the idea that geometrical judgments are carried out concurrently in both visual and haptic spaces (Hypothesis 3 ). When explored through only one of these modalities, the brain would nevertheless reconstruct representations of the task in the other and combine the results only after the spatial comparisons are carried out in each of these internal representations. The weight of each separate comparison on the final response would depend on the expected reliability of signals within each representation, which in turn depends on the brain's ability to transform and reconstruct signals between representations. Based on studies of goaldirected movements (Tagliabue and Mclntyre 2011, 2012, 2013, 2014, 2015, Tagliabue et al. 2013, Arnoux et al. 2017), gravity plays an important role in determining how reliably signals are transformed between sensory modalities, leading to predictable effects of removing gravity on shape perception. Out of the three possibilities presented here, logic and the quantitative assessments presented here support Hypothesis 3 as the most parsimonious explanation of the observed data. Furthermore, the structure of the concurrent model and the empirical results suggest a new hypothesis for future research, i.e. that the CNS may benefit from counter-balancing distortions within each modality to reduce errors when making geometric judgments in 3D space.

\section{Conclusions}

In the context of recent theories on optimal multi-sensory integration, our study provides a better understanding of human perception of 3D geometry. In particular, modifications of perception in microgravity provide fundamental cues to better understand how perception works "on Earth". We have provided here a unifying theory able to explain not only how gravity can influence an intrinsically egocentric process, but also the curious fact that gravity acts in opposite directions on haptic and visual perception. More importantly, our results extend the notion that gravity facilitates the transformation of information between concurrent, modality-specific internal representations of not only object orientation, but of object shape and size as well. 


\section{Bibliography}

Armstrong, L. \& Marks, L. E. (1999), 'Haptic perception of linear extent.', Perception \& psychophysics 61, $1211-1226$.

Arnoux, L.; Fromentin, S.; Farotto, D.; Beraneck, M.; Mclntyre, J. \& Tagliabue, M. (2017), 'The visual encoding of purely proprioceptive intermanual tasks is due to the need of transforming joint signals, not to their interhemispheric transfer.', Journal of neurophysiology 118, 1598--1608.

Avery, G. C. \& Day, R. H. (1969), 'Basis of the horizontal-vertical illusion.', Journal of experimental psychology $81,376-380$.

Barnett-Cowan, M.; Snow, J. C. \& Culham, J. C. (2015), 'Contribution of Bodily and Gravitational Orientation Cues to Face and Letter Recognition.', Multisensory research 28, 427-442.

Bourrelly, A.; McIntyre, J. \& Luyat, M. (2015), 'Perception of affordances during long-term exposure to weightlessness in the International Space station.', Cognitive processing 16 Suppl 1, 171-174.

Bourrelly, A.; Mclntyre, J.; Morio, C.; Despretz, P. \& Luyat, M. (2016), 'Perception of Affordance during ShortTerm Exposure to Weightlessness in Parabolic Flight.', PloS one 11, e0153598.

Buneo, C. A.; Jarvis, M. R.; Batista, A. P. \& Andersen, R. A. (2002), 'Direct visuomotor transformations for reaching.', Nature 416(6881), 632-636.

Burns, J. K. \& Blohm, G. (2010), 'Multi-sensory weights depend on contextual noise in reference frame transformations.', Front Hum Neurosci 4, 221.

Clément, G.; Vieville, T.; Lestienne, F. \& Berthoz, A. (1986), 'Modifications of gain asymmetry and beating field of vertical optokinetic nystagmus in microgravity.', Neuroscience letters 63, 271-274.

Clément, G. \& Eckardt, J. (2005), 'Influence of the gravitational vertical on geometric visual illusions.', Acta Astronaut 56(9-12), 911-917.

Clément, G.; Lathan, C. \& Lockerd, A. (2008), 'Perception of depth in microgravity during parabolic flight', Acta Astronautica 63(7), $828-832$.

Clément, G. \& Bukley, A. (2008), 'Mach's square-or-diamond phenomenon in microgravity during parabolic flight.', Neurosci Lett 447(2-3), 179-182.

Clément, G.; Fraysse, M.J. \& Deguine, O. (2009), 'Mental representation of space in vestibular patients with otolithic or rotatory vertigo.', Neuroreport 20, 457-461.

Clément, G. \& Reschke, M. F. (2010), Neuroscience in space, Springer Science \& Business Media.

Clément, G.; Skinner, A.; Richard, G. \& Lathan, C. (2012), 'Geometric illusions in astronauts during longduration spaceflight.', Neuroreport 23(15), 894-899.

Clément, G. \& Demel, M. (2012), 'Perceptual reversal of bi-stable figures in microgravity and hypergravity during parabolic flight.', Neuroscience letters 507, 143-146.

Clément, G.; Skinner, A. \& Lathan, C. (2013), 'Distance and Size Perception in Astronauts during LongDuration Spaceflight', Life 3(4), 524-537.

Clément, G.; Loureiro, N.; Sousa, D. \& Zandvliet, A. (2016), 'Perception of Egocentric Distance during Gravitational Changes in Parabolic Flight.', PloS one 11, e0159422.

Curry, R. E. (1972), A Bayesian Model for Visual Space Perception, in 'Seventh Annual Conference on Manual Control', pp. 187.

De Saedeleer, C.; Vidal, M.; Lipshits, M.; Bengoetxea, A.; Cebolla, A. M.; Berthoz, A.; Cheron, G. \& McIntyre, J. (2013), 'Weightlessness alters up/down asymmetries in the perception of self-motion.', Experimental brain research 226, 95-106.

Dupin, L.; Hayward, V. \& Wexler, M. (2018), 'Radial trunk-centred reference frame in haptic perception.', Scientific reports 8, 13550.

Dyde, R. T.; Jenkin, M. R. \& Harris, L. R. (2006), 'The subjective visual vertical and the perceptual upright.', Exp Brain Res 173(4), 612-622.

Ernst MO, Banks MS (2002) Humans integrate visual and haptic information in a statistically optimal fashion. Nature 415:429 - 433.

Fasse, E. D.; Hogan, N.; Kay, B. A. \& Mussa-Ivaldi, F. A. (2000), 'Haptic interaction with virtual objects. Spatial perception and motor control.', Biological cybernetics 82, 69-83.

Gentaz, E.; Baud-Bovy, G. \& Luyat, M. (2008), 'The haptic perception of spatial orientations.', Exp Brain Res 187(3), 331-348.

Girgus, J. S. \& Coren, S. (1975), 'Depth cues and constancy scaling in the horizontal-vertical illusion: the bisection error.', Canadian journal of psychology 29, 59-65. 
Goble, D. J. \& Brown, S. H. (2008), 'Upper limb asymmetries in the matching of proprioceptive versus visual targets.', J Neurophysiol 99(6), 3063-3074.

Goltz, H. C.; Irving, E. L.; Steinbach, M. J. \& Eizenman, M. (1997), 'Vertical eye position control in darkness: orbital position and body orientation interact to modulate drift velocity.', Vision research 37, 789-798.

Goodenough, D. R.; Oltman, P. K.; Sigman, E. \& Cox, P. W. (1981), 'The rod-and-frame illusion in erect and supine observers.', Perception \& psychophysics 29, 365-370.

Gurfinkel, V. S.; Lestienne, F.; Levik, Y. \& Popov, K. E. (1993), 'Egocentric references and human spatial orientation in microgravity. I. Perception of complex tactile stimuli.', Exp Brain Res 95(2), 339-42.

Hamburger, K. \& Hansen, T. (2010), 'Analysis of individual variations in the classical horizontal-vertical illusion.', Attention, perception \& psychophysics 72, 1045-1052.

Harris, L. R.; Jenkin, M.; Jenkin, H.; Dyde, R.; Zacher, J. \& Allison, R. S. (2010), 'The unassisted visual system on earth and in space.', J Vestib Res 20(1), 25-30.

Harris, L. R. \& Mander, C. (2014), 'Perceived distance depends on the orientation of both the body and the visual environment.', Journal of vision 14.

Henriques, D. Y.; Klier, E. M.; Smith, M. A.; Lowy, D. \& Crawford, J. D. (1998), 'Gaze-centered remapping of remembered visual space in an open-loop pointing task.', J Neurosci 18(4), 1583-1594.

Henriques, D. Y. P. \& Soechting, J. F. (2003), 'Bias and sensitivity in the haptic perception of geometry.', Experimental brain research 150, 95-108.

Howard, I. (1982), Human visual orientation, New York: Wiley.

Howard, I. P.; Bergström, S. S. \& Ohmi, M. (1990), 'Shape from shading in different frames of reference.', Perception 19, 523-530.

Kersten, D. \& Yuille, A. (2003), 'Bayesian models of object perception.', Current opinion in neurobiology 13, 150-158.

Kersten, D.; Mamassian, P. \& Yuille, A. (2004), 'Object perception as Bayesian inference.', Annu Rev Psychol $55,271-304$

Lee, T. S. (2015), 'The visual system's internal model of the world.', Proceedings of the IEEE. Institute of Electrical and Electronics Engineers 103, 1359-1378.

Lejeune, L.; Thouvarecq, R.; Anderson, D. I. \& Jouen, F. (2004), 'Kinesthetic estimation of the main orientations from the upright and supine positions.', Acta Psychol (Amst) 117(1), 13-28.

Leone, G. (1998), 'The effect of gravity on human recognition of disoriented objects.', Brain research. Brain research reviews 28, 203-214.

Lipshits, M. I.; Gurfinkel, E. V.; McIntyre, J.; Droulez, J.; Gurfinkel, V. S. \& Berthoz, A. (1994), Influence of weightlessness on haptic perception, in 'Life Sciences Research in Space', pp. 367.

Lipshits, M. \& McIntyre, J. (1999), 'Gravity affects the preferred vertical and horizontal in visual perception of orientation.', Neuroreport 10(5), 1085-1089.

Liu, Y.; Sexton, B. M. \& Block, H. J. (2018), 'Spatial bias in estimating the position of visual and proprioceptive targets.', Journal of neurophysiology 119, 1879-1888.

Loomis, J. M. \& Philbeck, J. W. (1999), 'Is the anisotropy of perceived 3-D shape invariant across scale?', Perception \& psychophysics 61, 397-402.

Loomis, J. M.; Klatzky, R. L. \& Giudice, N. A. (2013), Representing 3D space in working memory: Spatial images from vision, hearing, touch, and language 'Multisensory imagery', Springer, , pp. 131-155.

Luyat, M.; Gentaz, E.; Corte, T. R. \& Guerraz, M. (2001), 'Reference frames and haptic perception of orientation: body and head tilt effects on the oblique effect.', Percept Psychophys 63(3), 541-554.

Luyat, M. \& Gentaz, E. (2002), 'Body tilt effect on the reproduction of orientations: studies on the visual oblique effect and subjective orientations.', J Exp Psychol Hum Percept Perform 28(4), 1002-1011.

Marendaz, C.; Stivalet, P.; Barraclough, L. \& Walkowiac, P. (1993), 'Effect of gravitational cues on visual search for orientation.', Journal of experimental psychology. Human perception and performance 19, $1266-1277$.

McFarland, J. \& Soechting, J. F. (2007), 'Factors influencing the radial-tangential illusion in haptic perception.', Experimental brain research 178, 216-227.

McGuire, L. M. M. \& Sabes, P. N. (2009), 'Sensory transformations and the use of multiple reference frames for reach planning.', Nat Neurosci 12(8), 1056-1061.

McIntyre, J.; Stratta, F. \& Lacquaniti, F. (1997), 'Viewer-centered frame of reference for pointing to memorized targets in three-dimensional space.', J Neurophysiol 78(3), 1601-1618.

Mclntyre, J. \& Lipshits, M. (2008), 'Central processes amplify and transform anisotropies of the visual system in a test of visual-haptic coordination.', J Neurosci 28(5), 1246-1261. 
Paillard, J.J. Paillard, ed., (1991), Brain and Space, Oxford University Press, chapter Knowing where and knowing how to get there, pp. 461-481.

Prinzmetal, W. \& Beck, D. M. (2001), 'The tilt-constancy theory of visual illusions.', J Exp Psychol Hum Percept Perform 27(1), 206-217.

Reschke, M. F.; Kolev, O. I. \& Clément, G. (2017), 'Eye-Head Coordination in 31 Space Shuttle Astronauts during Visual Target Acquisition.', Scientific reports 7, 14283.

Reschke, M. F.; Wood, S. J. \& Clément, G. (2018), 'Ocular Counter Rolling in Astronauts After Short- and Long-Duration Spaceflight.', Scientific reports 8, 7747.

Smeets JBJ, van den Dobbelsteen JJ, de Grave DDJ, van Beers RJ, Brenner E (2006), Sensory integration does not lead to sensory calibration., Proc Natl Acad Sci USA 103(49): 18781-18786.

Tagliabue, M. \& McIntyre, J. (2011), 'Necessity is the Mother of Invention: Reconstructing Missing Sensory Information in Multiple, Concurrent Reference Frames for Eye-Hand Coordination.', J Neurosci 31(4), 1397-1409.

Tagliabue, M. \& McIntyre, J. (2012), 'Eye-hand coordination when the body moves: Dynamic egocentric and exocentric sensory encoding.', Neurosci Lett 513, 78-83.

Tagliabue, M.; Arnoux, L. \& Mclntyre, J. (2013), 'Keep your head on straight: Facilitating sensori-motor transformations for eye-hand coordination.', Neuroscience 248, 88-94.

Tagliabue, M. \& McIntyre, J. (2013), 'When Kinesthesia Becomes Visual: A Theoretical Justification for Executing Motor Tasks in Visual Space', PLoS ONE 8(7), e68438.

Tagliabue, M. \& Mclntyre, J. (2014), 'A modular theory of multisensory integration for motor control.', Front Comput Neurosci 8, 1.

Tagliabue M, Dal Canto D, Casadio M, McIntyre J. (2015) Effects of gravitational signals on visuo-kinesthetic sensory transformations during hand movements. Program No. 795.07. 2015 Neuroscience Meeting Planner. Washington, DC: Society for Neuroscience. Online.

Todd, J. T. \& Norman, J. F. (2003), 'The visual perception of 3-D shape from multiple cues: are observers capable of perceiving metric structure?', Perception \& psychophysics 65, 31-47.

van Beers, R. J.; Sittig, A. C. \& Gon, J. J. (1999), 'Integration of proprioceptive and visual positioninformation: An experimentally supported model.', J Neurophysiol 81(3), 1355-1364.

Vetter, P.; Goodbody, S. J. \& Wolpert, D. M. (1999), 'Evidence for an eye-centered spherical representation of the visuomotor map.', Journal of neurophysiology 81, 935-939.

Villard, E.; Garcia-Moreno, F. T.; Peter, N. \& Clément, G. (2005), 'Geometric visual illusions in microgravity during parabolic flight.', Neuroreport 16(12), 1395-1398.

Wagner, M. (1985), 'The metric of visual space', Perception \& psychophysics 38(6), 483-495.

Welchman, A. E. (2016), 'The Human Brain in Depth: How We See in 3D.', Annual review of vision science 2, 345-376.

Wolbers, T.; Klatzky, R. L.; Loomis, J. M.; Wutte, M. G. \& Giudice, N. A. (2011), 'Modality-independent coding of spatial layout in the human brain.', Current biology : CB 21, 984-989.

Wydoodt, P.; Gentaz, E. \& Streri, A. (2006), 'Role of force cues in the haptic estimations of a virtual length.', Experimental brain research 171, 481-489. 


\section{Appendix I: Quantifying Distortion}

As we stated in the methods section, it is not possible to univocally quantify the absolute distortion for each of the three dimensions, but only with respect to the other two dimensions. However, in order to provide in the results section a graphical representation of the perceptive distortions, the following method was used to compute the dimensional errors. We first solved the system of equations of Table 1 reported below in the matrix form.

$$
\left[\begin{array}{ccc}
1 & 0 & -1 \\
-1 & 0 & 1 \\
1 & -1 & 0 \\
-1 & 1 & 0 \\
0 & -1 & 1 \\
0 & 1 & -1
\end{array}\right] \cdot\left[\begin{array}{c}
\varepsilon_{E W} \\
\varepsilon_{N S} \\
\varepsilon_{U D}
\end{array}\right]=\left[\begin{array}{c}
\varepsilon_{E W-U D} \\
\varepsilon_{U D-E W} \\
\varepsilon_{E W-N S} \\
\varepsilon_{N S-E W} \\
\varepsilon_{U D-N S} \\
\varepsilon_{N S-U D}
\end{array}\right]
$$

If we call $A$ the matrix of linear coefficient, then the solutions of this underdetermined problem are

$$
\left[\begin{array}{c}
\varepsilon_{E W} \\
\varepsilon_{N S} \\
\varepsilon_{U D}
\end{array}\right]=A^{+} \cdot\left[\begin{array}{l}
\varepsilon_{E W-U D} \\
\varepsilon_{U D-E W} \\
\varepsilon_{E W-N S} \\
\varepsilon_{N S-E W} \\
\varepsilon_{U D-N S} \\
\varepsilon_{N S-U D}
\end{array}\right]+\left(I-A^{+} A\right) *\left[\begin{array}{c}
\varepsilon_{E W} \\
\varepsilon_{N S} \\
\varepsilon_{U D}
\end{array}\right]=A^{+} \cdot\left[\begin{array}{l}
\varepsilon_{E W-U D} \\
\varepsilon_{U D-E W} \\
\varepsilon_{E W-N S} \\
\varepsilon_{N S-E W} \\
\varepsilon_{U D-N S} \\
\varepsilon_{N S-U D}
\end{array}\right]+\left(I-A^{+} A\right) w=A^{+} \cdot\left[\begin{array}{l}
\varepsilon_{E W-U D} \\
\varepsilon_{U D-E W} \\
\varepsilon_{E W-N S} \\
\varepsilon_{N S-E W} \\
\varepsilon_{U D-N S} \\
\varepsilon_{N S-U D}
\end{array}\right]+\left[\begin{array}{l}
w \\
w \\
w
\end{array}\right]
$$

Where the $A^{\dagger}$ is the pseudo inverse of $A$ and $w$ is a free scalar parameter that reflects the fact that the observed results can be explained by an infinity of triplets of dimensional distortions differing by isotropic component, $w$, only (underdetermination of the problem).

For the representation of the data of Clément et al. (2008), given that 3 tasks (EW-NS, $U D-N S$ and NS-EW) were tested the $A$ matrix is

$A=\left[\begin{array}{ccc}1 & -1 & 0 \\ -1 & 1 & 0 \\ 0 & -1 & 1\end{array}\right]$

In all cases, to define a set of dimensional distortions, $\left(\varepsilon_{E W}, \varepsilon_{N S}, \varepsilon_{U D}\right)$ to be used for a graphical representation, we arbitrary decided to select the solution that minimizes the euclidean norm of the distortion vectors.

Although the $w$ parameter cannot be univocally defined, the difference between the errors along the three dimensions are correctly quantified and then used to test the anisotropy of the perceptive errors. The dimensional errors however cannot be rigorously compared between postures or gravitational conditions, because the differences between experimental conditions could be due to differences in defining the $w$ parameter for each condition. 


\section{Appendix II: Weighting based on Maximum Likelihood}

In order to compute the prediction about the effect of gravity on haptic and visual perception the following procedure has been used. Both visual and haptic comparisons, $\Delta$, between the adjustable and the reference size of the rectangle are characterized by a specific noisiness which is expressed in terms of variance. In the following table are reported the expressions of the variance of visual, $\Delta \mathrm{V}$, and haptic, $\Delta \mathrm{H}$, comparisons for both the visual and haptic task. The parameters that contribute to the two comparisons' variability are: the variance of haptic signals about the reference and adjustable sizes ( $\left.\sigma_{r e f, H}^{2}, \sigma_{a d j, H}^{2}\right)$ and the variance of the corresponding visual signals $\left(\sigma_{r e f, V}^{2}, \sigma_{a d j, V}^{2}\right)$; the variance associated to cross-modal transformations $\left(K^{2} \sigma_{t r}^{2}\right)$. This parameter includes a multiplying factor, $\mathrm{K}$, which is equal to 1 in normal gravity and which can increase in microgravity representing the possible difficulty of performing cross-modal transformation in microgravity. The last parameter is the variability associated to visual or haptic sensory systems metric $\left(\sigma_{m e t, V}^{2}, \sigma_{m e t, H}^{2}\right)$ allowing to compare length of stimuli differently oriented in space.

\begin{tabular}{l|l|l|}
\multirow{2}{*}{ Haptic task } & $\Delta \mathrm{V}$ & $\sigma_{\Delta V}^{2}=\left(\sigma_{r e f, H}^{2}+K^{2} \sigma_{t r}^{2}\right)+\left(\sigma_{a d j, H}^{2}+K^{2} \sigma_{t r}^{2}\right)+\sigma_{m e t, V}^{2}$ \\
\cline { 2 - 3 } & $\Delta \mathrm{H}$ & $\sigma_{\Delta H}^{2}=\sigma_{r e f, H}^{2}+\sigma_{a d j, H}^{2}+\sigma_{m e t, H}^{2}$ \\
\hline \multirow{2}{*}{ Visual task } & $\Delta \mathrm{V}$ & $\sigma_{\Delta V}^{2}=\sigma_{r e f, V}^{2}+\sigma_{a d j, V}^{2}+\sigma_{m e t, V}^{2}$ \\
\cline { 2 - 4 } & $\Delta \mathrm{H}$ & $\sigma_{\Delta H}^{2}=\left(\sigma_{r e f, V}^{2}+K^{2} \sigma_{t r}^{2}\right)+\left(\sigma_{a d j, V}^{2}+K^{2} \sigma_{t r}^{2}\right)+\sigma_{m e t, H}^{2}$
\end{tabular}

Following the maximum likelihood principle, for each task and condition the brain optimally combines the internal visual and haptic comparison in order to minimize the variability of the multi-sensory comparison, $\Delta$. The combination of the concurrent comparison can be expressed as $\Delta=W_{\Delta V} \Delta V+W_{\Delta H} \Delta H$ where each concurrent comparison is associated to an error due to the specific distortions of the corresponding sensory modality.

The optimal weight associated to each of the concurrent comparisons for both visual and haptic tasks are reported in the following table. The computation of these sensory weights takes into account the partial correlation between the concurrent comparisons (Tagliabue and Mclntyre 2013).

\begin{tabular}{l|l|l} 
Haptic task & $\Delta \mathrm{V}$ & $W_{\Delta V}=\frac{\sigma_{m e t, H}^{2}}{2 K^{2} \sigma_{t r}^{2}+\sigma_{m e t, V}^{2}+\sigma_{m e t, H}^{2}}$ \\
\cline { 2 - 3 } & $\Delta \mathrm{H}$ & $W_{\Delta H}=\frac{2 \sigma_{t r}^{2}+\sigma_{m e t, V}^{2}}{2 K^{2} \sigma_{t r}^{2}+\sigma_{m e t, V}^{2}+\sigma_{m e t, H}^{2}}$ \\
\hline \multirow{2}{*}{ Visual task } & $\Delta \mathrm{V}$ & $W_{\Delta V}=\frac{2 K^{2} \sigma_{t r}^{2}+\sigma_{m e t, H}^{2}}{2 K^{2} \sigma_{t r}^{2}+\sigma_{m e t, V}^{2}+\sigma_{m e t, H}^{2}}$ \\
\cline { 2 - 3 } & $\Delta \mathrm{H}$ & $W_{\Delta H}=\frac{\sigma_{m e t, V}^{2}}{2 K^{2} \sigma_{t r}^{2}+\sigma_{m e t, V}^{2}+\sigma_{m e t, H}^{2}}$
\end{tabular}


The variability of the corresponding optimal response for the haptic task is:

$\sigma_{\Delta_{H}}^{2}=W_{\Delta V}^{2} \sigma_{\Delta V}^{2}+W_{\Delta H}^{2} \sigma_{\Delta H}^{2}+2 \operatorname{cov}\left(W_{\Delta_{V}} \Delta_{V}, W_{\Delta_{H}} \Delta_{H}\right)=W_{\Delta V}^{2}\left(2 \sigma_{H}^{2}+2 K^{2} \sigma_{t r}^{2}+\sigma_{m e t}^{2}\right)+W_{\Delta H}^{2}\left(2 \sigma_{H}^{2}+\sigma_{m e t}^{2}\right)+2 W_{\Delta_{V}} W_{\Delta_{H}} 2 \sigma_{H}^{2}$ In order to reduce de number of parameters of the model few assumptions are made. First, for both visual and haptic modalities the noisiness associated to the adjustable and reference size of the rectangles is assumed to be the same:

$\sigma_{\text {ref }, V}^{2}=\sigma_{\text {adj, } V}^{2}=\sigma_{V}^{2}$

$\sigma_{r e f, H}^{2}=\sigma_{a d j, H}^{2}=\sigma_{H}^{2}$

The magnitude of the haptic perception variance has been computed from the results of Ernst and Banks (2002), obtained in a unidimensional haptic task and fixed to $\sigma_{H}^{2}=9 \mathrm{~mm}^{2}$ while the noise associated to the use of the sensory metric for the visual and haptic signals, although independent, has the same magnitude

$\sigma_{\text {met }, V}^{2}=\sigma_{\text {met }, H}^{2}=\sigma_{\text {met }}^{2}$

With these assumptions the model is characterized by 9 free internal parameters: $K, \sigma_{\text {tr }}^{2}, \sigma_{\text {met }}^{2},\left[\begin{array}{lll}\Delta_{\text {Sag }} & \Delta_{\text {Tra }} & \Delta_{\text {Fro }}\end{array}\right]_{V}$ and $\left[\begin{array}{lll}\Delta_{\text {Sag }} & \Delta_{\text {Tra }} & \Delta_{\text {Fro }}\end{array}\right]_{H}$.

The simulation consists in testing whether the model is able to reproduce the experimental results in normal and microgravity on haptic perception presented here in terms of both constant errors $\left(\left[\begin{array}{lll}\Delta_{\text {Sag }} & \Delta_{\text {Tra }} & \Delta_{\text {Fro }}\end{array}\right]_{1 G, H},\left[\begin{array}{lll}\Delta_{\text {Sag }} & \Delta_{\text {Tra }} & \Delta_{F r o}\end{array}\right]_{0 G, H}\right)$ and variability ( $\sigma_{\Delta_{1 G, H}}^{2}, \sigma_{\Delta_{0 G, H}}^{2}$ ); as well as the results reported by Clément et al. 2008 for the constant error of visual perception $\left(\left[\begin{array}{lll}\Delta_{S a g} & \Delta_{T r a} & \Delta_{F r o}\end{array}\right]_{1 G, V},\left[\begin{array}{lll}\Delta_{S a g} & \Delta_{T r a} & \Delta_{F r o}\end{array}\right]_{0 G, V}\right)$. No data about response variability is reported for visual perception.

The identification of the parameter that best reproduce the experimental data has been performed using an optimization algorithm (Matlab fmincon function) minimizing the difference between the model predictions and the experimental results. 